\title{
Cross-impact analysis experimentation using two techniques to revise marginal probabilities of interdependent events
}

\author{
MI Mphahlele* OO Olugbara* $\quad$ SO Ojo* $\quad$ DG Kourie ${ }^{\dagger}$ \\ Received: 30 August 2010; Revised: 12 March 2011; Accepted: 22 March 2011
}

\begin{abstract}
Cross-impact analysis relies on decision makers to provide marginal probability estimates of interdependent events. Generally, these have to be revised in order to ensure overall system coherency. This paper describes cross-impact analysis experimentation in which a Monte Carlo based approach and a difference equation approach, respectively, were used to revise these marginal probabilities. The objective of the study was to determine the consequences of such revisions on the expected impact rankings of these events. A cross-impact analysis system was developed and used to conduct the experiments. The experiments show that the impact ranking of interdependent events may indeed depend on the technique used for revising event marginal probabilities. Moreover, the Monte Carlo technique generates a world view closer to the one of the decision makers, while the world view generated by the difference equation technique differs from that of the decision makers.
\end{abstract}

Key words: Cross-impact analysis, probability revision, monte carlo simulations, difference equations.

\section{Introduction}

The government of the Republic of South Africa, like other governments across the world, has realised the importance of information technology for economic growth. Consequently, a drive towards developing e-skills programs has gained momentum. A working group comprising of industry representatives, government agents and academy was inaugurated by the Minister of Communication to address, among others, an e-skills strategy that will bring about e-skills development. It is in the light of this initiative that the working group identified scenario analysis methods - a forecasting exercise - as a tool to support the formulation of an e-skills strategy for the country.

The process of forecasting futures over a time horizon of say 10 to 15 years, requires decision makers and experts to have access to possible alternative futures from which to

\footnotetext{
*Department of Computer Networks, Tshwane University of Technology, Pretoria, South Africa

${ }^{\dagger}$ Corresponding author: Espresso Research Group, Department of Computer Science, University of Pretoria, Pretoria, South Africa, email: dkourie@cs.up.ac.za
} 
make choices. The main aim of futures studies is to systematically explore, create and analyse probable future developments to enhance decisions taken for the future [1]. If the future could be determined by the past and present, futures research would have been a definite exercise.

Unfortunately, unprecedented events [8] also do play a significant role in shaping the future, and it is required of us to account for these unprecedented events in exploring possible futures. Moreover, the evolution of the future is shaped by controllable events such as social, economic, political, cultural, environmental and technological actions. It is, therefore, essential that decision makers understand the full range of possible alternatives in order to improve the decision making process. Cross-Impact Analysis (CIA) and scenario generation enable decision makers to explore alternative futures [8].

This article reflects work that was undertaken by members of the aforementioned working group. The purpose was to gain a sense of the validity of CIA as a scenario analysis instrument in terms of its robustness and sensitivity to data inputs. We believe that the results are of general interest to those who use or might potentially use the method. Further work based on live data is currently underway as part of the working group's brief, and will be reported once completed and publication clearance has been obtained.

Among the important highlights of CIA is its ability to establish a ranking of interdependent events. Such a ranking could either be from least to most likely to occur, or - as in the present case - from least to highest expected impact. Event ranking relies on the determination of a full range of important impacts resulting from interactions between interdependent events. Event interactions reveal causal linkages in terms of the level at which an occurrence of one event is expected to increase or decrease the likelihood of occurrence of other events.

Generally, the CIA methodology entails the identification of crucial causal linkages, as well as the execution of sensitivity analysis to establish the impact of possible alternative developments and to reveal insignificant events that are to be isolated. The types of sensitivity analysis may be with respect to (a) the impact of the occurrence of certain events, (b) the impact of the non-occurrence of certain events and (c) the impact of policy changes [7].

The successful application of sensitivity analysis requires the ranking of events. By highlighting events that play a significant role, decision makers are enabled to systematically explore, create and analyse probable futures. The testing for possible alternative futures involves the evaluation of direct change of probabilities and causal interplay that takes place among events. The strength of a CIA technique is its ability to take cognisance of the impacts that separate components may have upon one another [11] and to reveal critical events that are actors or reactors within those components. This makes CIA techniques considerably different from older techniques such as Delphi [3] for future forecasting.

In this paper, we report on CIA experimentation in which two techniques are used to revise marginal probabilities of interdependent events. The first technique is based on Monte Carlo simulation, and the second on the difference equation approach pioneered by Turoff [14]. The objective of the study was to determine the effect of marginal probabilities revision on the ranking of interdependent events in terms of their probable (or expected) 
impact. The remainder of the paper is organised as follows. In Section 2, we summarise related work. In Section 3, we describe the methodology to build a cross-impact analysis system that was used to conduct the CIA experimentation. In Section 4, we describe the experimentation to determine the effects of marginal probabilities revision on the ranking of interdependent events. In Section 5, we give a brief conclusion of the paper.

\section{Related work}

There are several variants of CIA techniques. In general they involve decision maker estimates of marginal probabilities of interdependent events, and possibly also estimates of conditional probabilities of these events.

The marginal probability of an event designates the probability that it will occur - i.e. without regard to the influence that the occurrence of other events might have on the probability of this event's occurrence. The conditional probability of an event estimates the event's occurrence probability, subject to the occurrence of some other event.

Some CIA systems also make use of estimates of cross-impact factors in relation to these interdependent events. A cross-impact factor of an event is an estimation of the level and/or direction of impact that the event will make if and when it occurs. These factors are generally recorded in a matrix in which a cell entry designates the impact that a column-specified event is estimated to have on a row-specified event.

Various systems require decision makers to supply various sets of inputs. Examples include (a) event marginal probabilities, occurrence conditional probabilities and non-occurrence conditional probabilities [14]; (b) event marginal probabilities, occurrence conditional probabilities and a causal cross-impact matrix [2]; and (c) event marginal probabilities, occurrence conditional probabilities and times of impartation [1]. Keeping the number of inputs solicited from decision makers to a minimum can definitely help to (a) improve system prediction accuracy, (b) minimize input errors and (c) save decision makers from the rigour of having to simultaneously supply many inputs.

There are many CIA techniques $[4,10,14]$ that have proposed the revision of the marginal probabilities of events. Some other CIA techniques [1,2] consider revising the cross-impact matrix, instead of the marginal probabilities of events, in order to improve the accuracy of ranking events. Other CIA techniques $[1,9,13]$ considered event ranking by classifying interdependent events into influential, dependent, key, excluded and neutral categories of events.

In this paper the important reason why we have to revise inputs supplied by decision makers is to eliminate possible inconsistencies. For example, inconsistencies can naturally occur between marginal and conditional probabilities of events, thereby violating the fundamental laws of probability calculus. For decision makers, it is practically impossible to correctly enumerate all possible causal interactions between events to reflect the world views of the modelled system.

This study considers ways of revising event marginal probabilities assumed to have been provided by decision makers, and determines the effect this revision has on the ranking of 
events in terms of expected impact. We keep the number of inputs supplied by decision makers to a minimum by allowing two types of inputs, namely marginal probabilities and a causal cross-impact matrix. Since the expected impact depends on conditional probabilities (as well as on the original cross-impact matrix) we also have to update these probabilities whenever the marginal probabilities are changed. The details of this methodology are discussed in the next section.

\section{Methodological design}

We assume that decision makers have identified a set of relevant events, together with an estimate of the marginal probability that these events will occur, as well as a causal cross-impact matrix. Our subsequent methodology then involves four computational steps, discussed in the next four subsections respectively:

- computation of revised marginal probabilities,

- computation of consistent occurrence conditional probabilities,

- computation of a likely causal cross-impact matrix or a revised causal cross-impact matrix, and

- ranking of events.

Figure 1 illustrates the overall architecture of our CIA system that was used for the experimentation. At the higher level, the system receives two forms of inputs from the decision makers, namely marginal probabilities and a causal cross-impact matrix. The output of the system is a ranking of scores computed with regard to the probable (expected) impact of the various events. At the lower levels, the inputs supplied by the decision makers are taken through the four computational steps to realise the output.

\subsection{Computation of revised marginal probabilities}

The first computational step is to calculate revised marginal probabilities from the marginal probabilities and causal cross-impact matrix supplied by the decision makers. The Monte Carlo and difference equation techniques are separately employed to revise marginal probabilities of events.

\subsubsection{The Monte Carlo approach}

The Monte Carlo approach $[4,10,11]$ comprises the following:

(a) Random selection of an event to test for its occurrence or non-occurrence.

(b) Random selection of a real number in the interval $[0,1]$ to compare with the estimated marginal probability of the randomly selected event to test for occurrence. If the probability of the randomly selected event is greater than the randomly selected real number, then the event is considered to have occurred; otherwise the event is considered not to have occurred. 


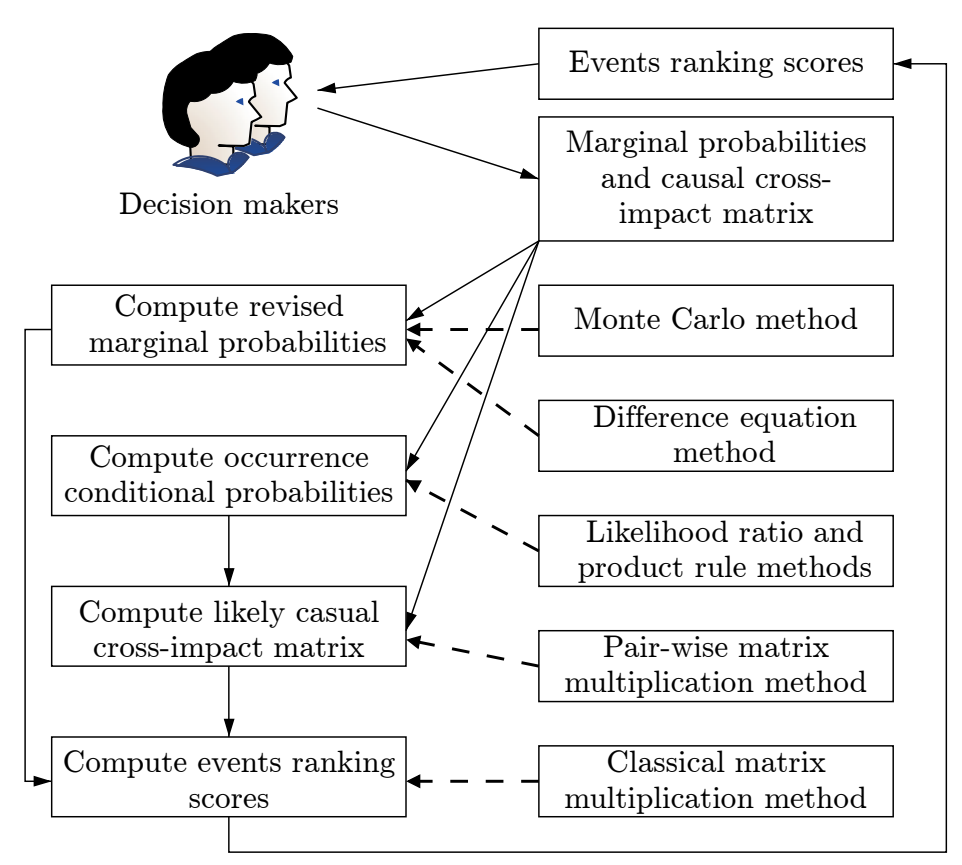

Figure 1: Architecture of the CIA system used in this paper.

(c) The probabilities of the remaining events are revised depending on whether the selected event has or has not occurred. The way in which this revision happens is briefly explained below.

(d) Step (a) - (c) are repeated until each event is tested for occurrence or non-occurrence in each play.

All the probabilities are then returned to their initial values and this entire process is replayed 500 times [11]. The relative frequency of occurrence of each event for all plays determines the revised probability of the event.

The probability revision required in step (c) above requires further explanation. We note that if it is determined in step (b) that event $j$ has occurred, then it is reasonable to assume that the new probability of event $i$ occurring in the next Monte Carlo iteration is provided by an estimate of the conditional probability, $p(i \mid j)$. Similarly, if it is determined in step (b) that event $j$ has not occurred, then the conditional probability $p(i \mid \bar{j})$ of $i$ occurring if $j$ did not occur serves as a new estimate of $p(i)$ in the next Monte Carlo iteration. A more detailed discussion of how these conditional probabilities may be computed from current probability estimates and from cross-impact data follows in the next section. Note that the computation of conditional probabilities is discussed separately, because such computation is a necessary step after the computation of revised marginal probability estimates, whether by a Monte Carlo approach as discussed above, or by the difference equation method, as discussed next. 


\subsubsection{The difference equation method}

The second method that we investigated for revising marginal probabilities of interdependent events is based on the difference equation method [14]. The method assumes the existence of a set of $n$ equations expressing each of the probabilities of $n$ events as a function of the $n-1$ probabilities. Thus each difference equation is enunciated as

$$
p(i)=p_{i}(p(1), p(2), \ldots, p(i-1), p(i+1), \ldots, p(n)) .
$$

The set of difference equations may also include variables expressing causal interactions of potential events not specified by the event list. If an individual estimator receives new information that requires a change in his/her estimates for any of the marginal probabilities, the change is consistent with the difference equation (1). Hence this equation can be written as the boundary value problem

$$
\delta p(i)= \begin{cases}0 & \text { if } p(i)=0 \text { or } p(i)=1 \\ \sum_{k \neq i}^{n} \frac{\partial p(i)}{\partial p(k)} \delta p(k)+\frac{\partial p(i)}{\partial \beta} \delta \beta & \text { otherwise }\end{cases}
$$

where $\beta$ is considered as a collective measure of the impact of those events that are not included in the specified set.

The difference equation approach [14] is a so-called cascading perturbation-based technique. In each iteration, or cascade, the event whose marginal probability is closest to one or zero is determined - i.e. the event most likely to occur or not occur, as the case may be. The event's current marginal probability is then stored as that event's final marginal probability. However, for perturbation purposes, the event is assumed to have occurred / not occurred. Based on this assumption, new marginal probabilities of the remaining events are computed. These "perturbations" are continued until the outcome is established for all events.

In each iteration, the probability $p(i)$ of the $i^{\text {th }}$ event is recalculated using the Fermi-Dirac type of equation,

$$
p(i)=\frac{1}{1+\exp \left(-\gamma_{i}-\sum_{i \neq k}^{n} c_{i k} p(k)\right)},
$$

which is a solution to (2). Here $\gamma_{i}$ may be interpreted as a term that accounts for the impact of unknown external events on event $i$. Each parameter $\gamma_{i}$ is a function of original or unrevised marginal probabilities of events and the causal cross-impact matrix. It is computed as

$$
\gamma_{i}=\phi(p(i))-\sum_{k \neq i}^{n} c_{i k} p(k)
$$

where

$$
\phi(p(i))=\ln \left(\frac{p(i)}{1-p(i)}\right)
$$

is a measure of occurrence ratio for expressing the likelihood of the occurrence of an event. 


\subsection{Computation of consistent conditional probabilities}

The second step of the computation is to compute consistent occurrence conditional probabilities of events. The so-called constant likelihood ratio method described in Duval et al. [4] is used to accomplish this computational task.

Using this method, the conditional probability $p(i \mid j)$ of the $i^{\text {th }}$ event given that the $j^{\text {th }}$ event has occurred, is calculated from the likelihood ratio, $r_{i j}$, as

$$
p(i \mid j)=\frac{r_{i j} p(i)}{1+\left(r_{i j}-1\right) p(i)} .
$$

The value of $r_{i j}$ is derived from the corresponding cross-impact factor $c_{i j}$ via the piecewise equation

$$
r_{i j}= \begin{cases}\frac{(r-1) c_{i j}+a}{a} & \text { if } c_{i j} \geq 0 \\ \frac{a}{(1-r) c_{i j}+a} & \text { if } c_{i j}<0 .\end{cases}
$$

Here it is assumed that cross-impact factor estimates are based on a scale in the interval $[-a, a]$. Generally the parameter $a$ is chosen in the interval $[1,10]$, varying according to the needs of the study concerned. Similarly, the parameter $r$ can be any value larger than 0 . Its choice fixes the range of likelihood ratio values as $\left[\frac{1}{r}, r\right]$.

Figure 2 illustrates the relationship between the constant likelihood ratio, $r_{i j}$, and the cross-impact factor, $c_{i j}$, in (3). The value for $a$ used in Figure 2 is 5 , and various graphs for $r=4,6,8,12$ have been plotted. The non-linear relationship between $r_{i j}$ and $c_{i j}$ is such that $r_{i j}$ remains in the interval $\left[\frac{1}{r}, r\right]$. Note that a positive cross-impact factor indicates that a driving event enhances a driven event, and results in a high likelihood ratio. Conversely, a negative cross-impact factor indicates that the driving event inhibits the driven event and is associated with a likelihood ratio close to 0 .

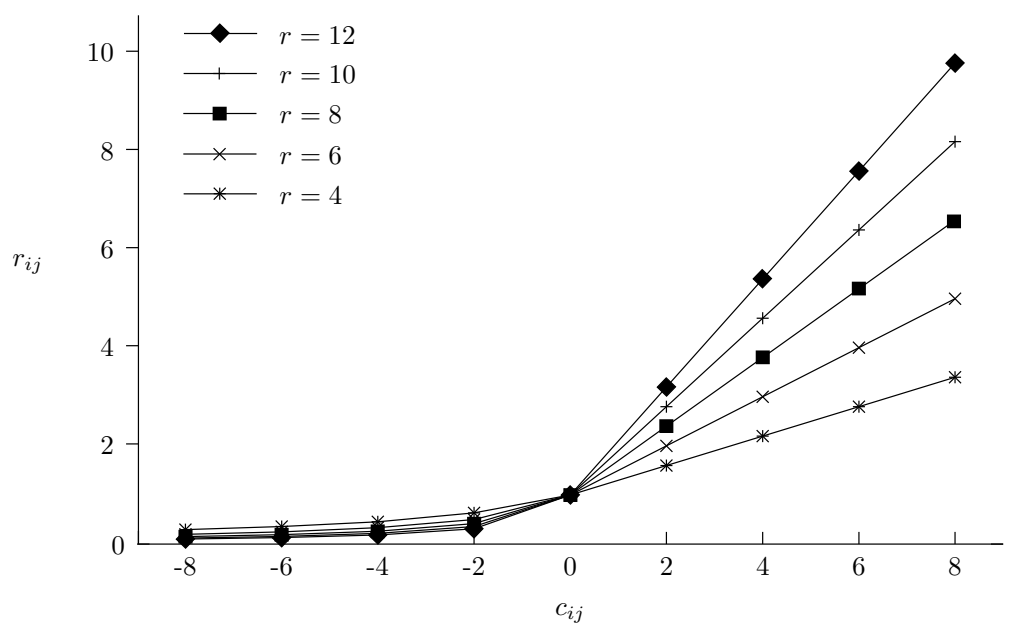

Figure 2: Relationship between the likelihood ratio and cross-impact factor.

The mapping of a cross-impact factor described by (3) reflects the symmetry of the relationship between the conditional probability and the marginal probability based on the 
likelihood ratio. This symmetric relationship can be observed by plotting the graph of conditional probability against marginal probability for a particular $r$ value. An example can be found in Duval et al [4].

Note that the computed value of $p(i \mid j)$ by the likelihood ratio method, as shown in (3), can fall outside the acceptable range of $[0,1]$ for a probability [6]. This problem can arise if the computed conditional probability $p(i \mid j)$ value violates the $[0,1]$ range on probabilities, or if the conditional and marginal probabilities, taken together, violate Bayes' Theorem. Consequently, it is required that

$$
p(i \mid j)^{-} \leq p(i \mid j) \leq p(i \mid j)^{+}
$$

where the model of De Kluyver and Moskowitz (cited in Nguyen and Dunn [12]) provides the bounds

$$
\begin{aligned}
& p(i \mid j)^{-}=\max \left\{0, \frac{p(i)+p(j)-1}{p(j)}\right\} \text { and } \\
& p(i \mid j)^{+}=\min \left\{1, \frac{p(i)}{p(j)}\right\}
\end{aligned}
$$

on the conditional probabilities. (Note that Sarin's model (also cited in Nguyen and Dunn [12]) gives similar bounds on joint probabilities.)

If the limit constraint is violated, the $p(i \mid j)$ value is replaced by the mean of the probability limits to achieve consistency between conditional and marginal probabilities. Thus, it follows that

$$
p(i \mid j)=\frac{p(i \mid j)^{-}+p(i \mid j)^{+}}{2} .
$$

The mean of a data sample as a measure of central tendency is a candidate representation of the sample.

A consistent occurrence conditional probability $p(i \mid j)$ of an interdependent event given that another event has occurred can be computed using the formulae

$$
\begin{aligned}
& p(i \mid j)= \begin{cases}\frac{r_{i j} p(i)}{1+\left(r_{i j}-1\right) p(i)} & \text { if } i<j \\
1 & \text { if } i=j, \quad \text { and }\end{cases} \\
& p(j \mid i)=\frac{r_{i j} p(j)}{1+\left(r_{i j}-1\right) p(i)} \quad \text { if } i>j .
\end{aligned}
$$

The same procedure of validating the computed value of occurrence conditional probability is applied when (5) to (6) are used to calculate occurrence conditional probabilities. The occurrence conditional probability is then estimated via (4) if the limit constraint is violated. It is easy to verify that the likelihood ratio method described by (5) and (6) for estimating the occurrence conditional probability give a higher enhancing or inhibiting effect than the mean limit method that (4) describes.

\subsection{Computation of likely causal cross-impact matrix}

The third step of the computation is to calculate the likely causal cross-impact matrix using pair-wise matrix multiplication [2]. The likely cross-impact matrix $L(i, j)=\left[\ell_{i j}\right]$ 
is formed by multiplying each element of the occurrence conditional probability matrix $P(i, j)$ by the corresponding element of the causal cross-impact matrix $C(i, j)$. Thus, it follows that

$$
\left(\begin{array}{cccc}
\ell_{11} & \ell_{12} & \cdots & \ell_{1 n} \\
\ell_{21} & \ell_{22} & \cdots & \ell_{2 n} \\
\vdots & \vdots & \ddots & \vdots \\
\ell_{n 1} & \ell_{n 2} & \cdots & \ell_{n n}
\end{array}\right)=\left(\begin{array}{cccc}
p(1 \mid 1) c_{11} & p(1 \mid 2) c_{12} & \cdots & p(1 \mid n) c_{1 n} \\
p(2 \mid 1) c_{21} & p(2 \mid 2) c_{22} & \cdots & p(2 \mid n) c_{2 n} \\
\vdots & \vdots & \ddots & \vdots \\
p(n \mid 1) c_{n 1} & p(n \mid 2) c_{n 2} & \cdots & p(n \mid n) c_{n n}
\end{array}\right)
$$

\subsection{Ranking of interdependent events}

The final step of the computation is to rank interdependent events using classical matrix multiplication. Using the likely and expected impact method [2], each element $e_{i}$ of the expected impact vector $E(i)=\left[e_{i}\right] \in \mathbb{R}^{n}$ is calculated from the likely cross-impact matrices, $L(i, j)$, and from the revised marginal probabilities of events $P(i)$ to give the ranking score $e_{i}(i=1,2, \ldots, n)$ of each event as follows.

$$
\left(\begin{array}{c}
e_{1} \\
e_{2} \\
\vdots \\
e_{n}
\end{array}\right)=\left(\begin{array}{cccc}
\ell_{11} & \ell_{12} & \ldots & \ell_{1 n} \\
\ell_{21} & \ell_{22} & \ldots & \ell_{2 n} \\
\vdots & \vdots & \ddots & \vdots \\
\ell_{n 1} & \ell_{n 2} & \ldots & \ell_{n n}
\end{array}\right)\left(\begin{array}{c}
p(1) \\
p(2) \\
\vdots \\
p(n)
\end{array}\right)
$$

Note that the net expected impact can be obtained from equation (8) by summing all the computed ranking scores - i.e. by summing the expected impacts of all events.

\section{Implementation and discussion of results}

The purpose of the experimentation is to determine the effect of the revision of marginal probabilities on the ranking of interdependent events. Two numerical examples considered for the experimentation are a $3 \times 3$ cross-impact matrix system taken from [2] and a $10 \times 10$ cross-impact matrix system taken from [5]. Tables 1 and 2 show the causal crossimpact matrices in which a driving event (column) is estimated to impact - positively or negatively - on a driven event (row) to the extent indicated in the corresponding cell ${ }^{1}$.

At the bottom of each table, the originally estimated marginal probabilities of the respective events are also provided. The value of $a$ for the $3 \times 3$ matrix is 5 , while for the $10 \times 10$ matrix, it is 3. Throughout this experiment the $r$ value used in (3) was 10 .

\footnotetext{
${ }^{1}$ The authors of the two texts from which these respective systems were taken ascribe different interpretations to the diagonal entries. In the $3 \times 3$ case, the author proposes that a diagonal entry should represent the overall impact of the relevant event on the system under study. In the $10 \times 10$ case, the more traditional interpretation is rendered, namely that an event's occurrence should be viewed as vacuously having no impact on its occurrence. These differing interpretations have no material bearing on the results of the present study.
} 


\begin{tabular}{cccc}
\hline Events & 1 & 2 & 3 \\
\hline 1 & -5 & -3 & 2 \\
2 & 2 & 3 & 4 \\
3 & -2 & 2 & 5 \\
\hline$P(i)$ & 0.70 & 0.50 & 0.40 \\
\hline
\end{tabular}

Table 1: The $3 \times 3$ causal cross-impact matrix, where the column events (driving events) affect the row events (driven events).

\begin{tabular}{ccccccccccc}
\hline Events & 1 & 2 & 3 & 4 & 5 & 6 & 7 & 8 & 9 & 10 \\
\hline 1 & 0 & 0 & 0 & 1 & 1 & -1 & 0 & -1 & 0 & 1 \\
2 & 0 & 0 & 1 & -1 & 0 & -1 & 1 & -1 & 0 & 0 \\
3 & 1 & 2 & 0 & 0 & 1 & 0 & 1 & 0 & 1 & 1 \\
4 & 1 & 0 & 0 & 0 & 1 & 3 & 0 & -1 & 0 & 1 \\
5 & 2 & 0 & 1 & 2 & 0 & 2 & 0 & 1 & 1 & 0 \\
6 & 1 & 0 & 0 & 2 & 2 & 0 & 0 & -1 & 0 & 1 \\
7 & 0 & 0 & 0 & 0 & 0 & 0 & 0 & 0 & 0 & 0 \\
8 & -1 & 0 & 0 & 0 & 0 & 1 & 1 & 0 & 0 & 1 \\
9 & 1 & 1 & 1 & 1 & 1 & 1 & -1 & 1 & 0 & 1 \\
10 & 1 & 1 & 1 & 1 & 1 & 1 & 0 & 1 & 0 & 0 \\
\hline$P(i)$ & 0.80 & 0.10 & 0.50 & 0.50 & 0.60 & 0.60 & 0.23 & 0.50 & 0.30 & 0.25 \\
\hline
\end{tabular}

Table 2: The $10 \times 10$ causal cross-impact matrix, where the column events (driving events) affect the row events (driven events).

\subsection{Ranking order experiments}

Table 3 shows the original Unrevised (UNR), Monte Carlo Revised (MCR) and Difference Equation Revised (DER) marginal probabilities of events 1, 2 and 3.

\begin{tabular}{cccc}
\hline & $P(1)$ & $P(2)$ & $P(3)$ \\
\hline UNR & 0.70 & 0.50 & 0.40 \\
MCR & 0.66 & 0.57 & 0.40 \\
DER & 0.82 & 0.12 & 0.40 \\
\hline
\end{tabular}

Table 3: Marginal probabilities for the $3 \times 3$ system.

In terms of these probabilities, the world view generated by the Monte Carlo technique is close to the world view of the decision makers. However, there is a clear difference between the world view generated by the difference equation method and the world view of the decision makers; in terms of rankings, event 2 is adjudged the least probable by the difference equation method, but not by the decision makers.

After revising the conditional probabilities as described in $\S 3.2$, these probabilities were then used in equation (7) to estimate the revised cross-impact factors, $\ell_{i j}$. In turn, these were used in equation (8) to compute the the expected impact values $(e(1), e(2)$ and $e(3))$ of the interdependent events in the $3 \times 3$ system.

In Figure 3 these expected impact values, resulting from the Monte Carlo (MC), the original, and the difference equation (DE) probabilities, respectively, are ranked. There is no difference in the ranking orders generated for this smaller system; in each case, event $e(2)$ has the highest rank, followed by $e(3)$, followed by $e(1)$. 
MC Revised Probability Original Probability DE Revised Probability

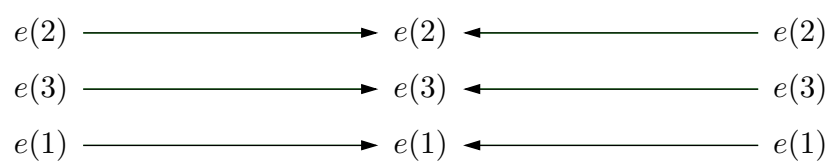

Figure 3: Comparison of expected impact ranking order for a $3 \times 3$ system.

\begin{tabular}{ccccccccccc}
\hline & $P(1)$ & $P(2)$ & $P(3)$ & $P(4)$ & $P(5)$ & $P(6)$ & $P(7)$ & $P(8)$ & $P(9)$ & $P(10)$ \\
\hline UNR & 0.80 & 0.10 & 0.50 & 0.50 & 0.60 & 0.60 & 0.23 & 0.50 & 0.30 & 0.25 \\
MCR & 0.72 & 0.14 & 0.66 & 0.66 & 0.76 & 0.68 & 0.24 & 0.52 & 0.63 & 0.62 \\
DER & 0.13 & 0.10 & 0.22 & 0.06 & 0.06 & 0.29 & 0.21 & 0.70 & 0.27 & 0.26 \\
\hline
\end{tabular}

Table 4: Marginal probabilities for the $10 \times 10$ system.

This entire exercise was then repeated for the larger $10 \times 10$ system. Table 4 gives original decision makers (UNR), Monte Carlo-computed (MCR) and difference equation-computed (DER) marginal probabilities of the events $1,2, \ldots 10$. Inspection of the values suggests that the probability world view generated by the MCR method is fairly close to that of UNR. On the other hand, the probability world view generated by the DER method appears to be rather different from UNR. These impressions about the probability rankings can be confirmed and quantified by computing Spearman's rank correlation coefficient, $\rho$. As seen in the first row of Table $5, \rho(\mathrm{MCR}, \mathrm{UNR})$ in the case of the probability data is 0.988 - a value so close to 1 that the rankings are almost identical. On the other hand, $\rho$ (DER, UNR) with respect to probability data comes to 0.697 , which weakly suggests a positive correlation in the probability data.

\begin{tabular}{lcc}
\hline & MCR & DER \\
\hline Original vs Revised $P$ & 0.988 & 0.697 \\
Original vs Revised $e$ & 0.919 & -0.191 \\
\hline
\end{tabular}

Table 5: Spearman's rank correlation coefficient, $\rho$, for the $10 \times 10$ system.

Again, using equation (8), the expected impact values $(e(1), e(2) \ldots e(10))$ of the interdependent events in the $10 \times 10$ system were computed. Figure 4 visually represents the rankings of these expected impact values, and the second row of Table 5 gives Spearman's rank correlation coefficient, $\rho$, for this data. It shows that the expected impact rankings derived from the Monte Carlo-revised probabilities remain very strongly positively correlated with the rankings based on the original probabilities $(\rho(\mathrm{MCR}, \mathrm{UNR})=0.919)$. On the other hand, the rankings derived from the different equation probability revisions show practically no correlation whatsoever with the original rankings $(\rho(\mathrm{DER}, \mathrm{UNR})=-0191)$.

Clearly, therefore, in this $10 \times 10$ system, the ranking orders generated by the two methods differ somewhat with respect to original versus revised probabilities, and the ranking orders differ decisively with respect to original versus revised expected impact. 
MC Revised Probability Original Probability DE Revised Probability

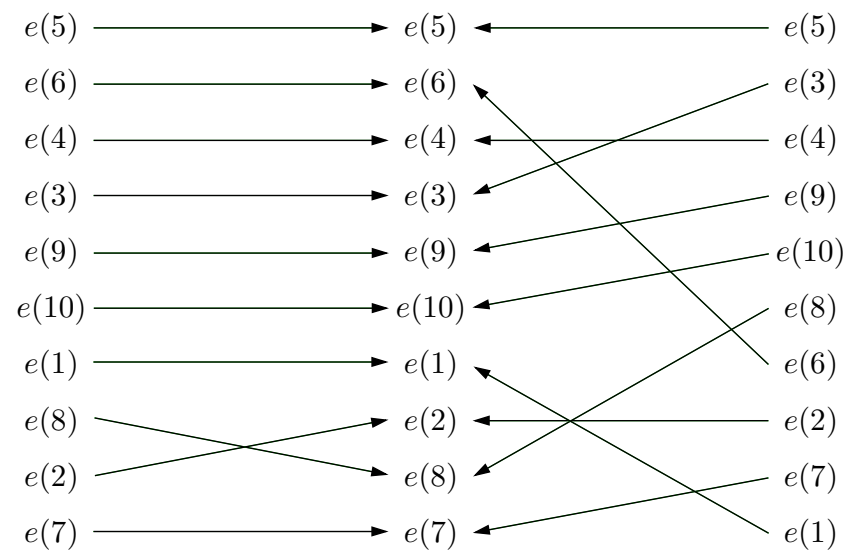

Figure 4: Comparison of expected impact ranking order for the $10 \times 10$ system.

\subsection{Sensitivity of initial probability estimates}

The Mean Net Expected Impact (MNEI) is the mean of the net expected impacts over all events, given by $\sum_{i=1}^{n} e(i) P(i)$. A sensitivity experiment was conducted to determine the impact of change in probability (as input variable) on MNEI (as output variable). This was done by selecting the most influential event and varying its event probability in the range of 0.1 to 1 while keeping probabilities of the remaining $(n-1)$ events fixed.

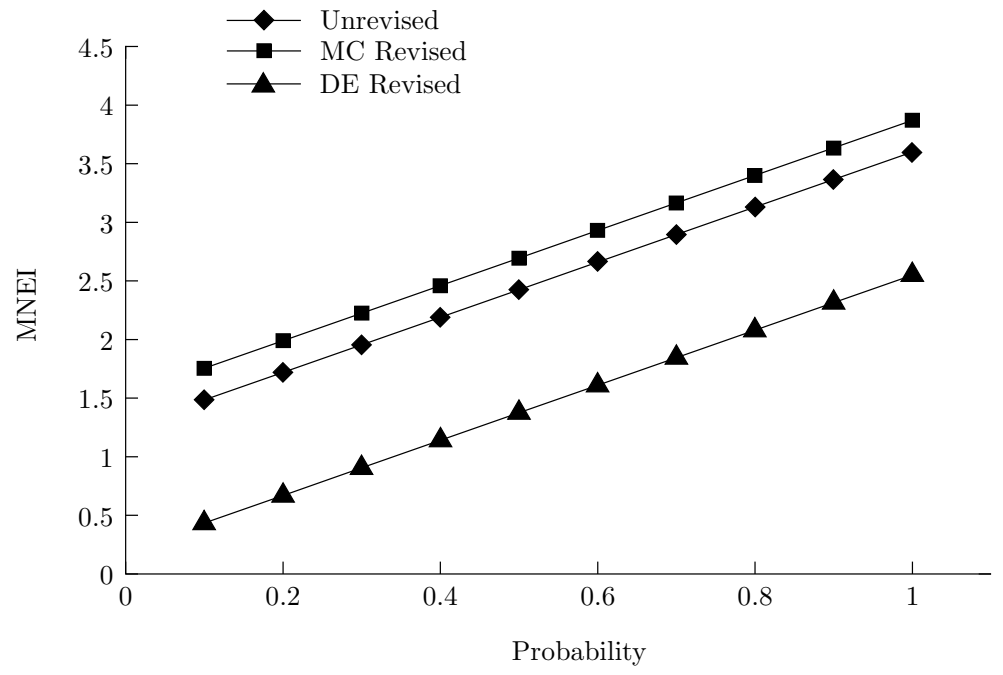

Figure 5: Relationship between MNEI and probability for the $3 \times 3$ system.

Figure 5 shows the result of the sensitivity experiment to determine the impact of probabilities revision on the ranking of interdependent events for the case of a $3 \times 3$ system. The result shows a linear relationship between MNEI against probability. It can be observed from the figure that the graph of MNEI computed from unrevised marginal probability against probability is closer to the graph of MNEI computed from Monte Carlo revised probability against probability, but farther from the graph of MNEI computed from dif- 
ference equation revised probability against probability.

Figure 6 shows the result of the sensitivity experiment for the case of a $10 \times 10$ system. The result also shows a linear relationship between MNEI against probability. It can also be observed from the figure that the graph of MNEI computed from unrevised marginal probability against probability is closer to the graph of MNEI computed from Monte Carlo revised probability against probability, but farther from the graph of MNEI computed from difference equation revised probability against probability.

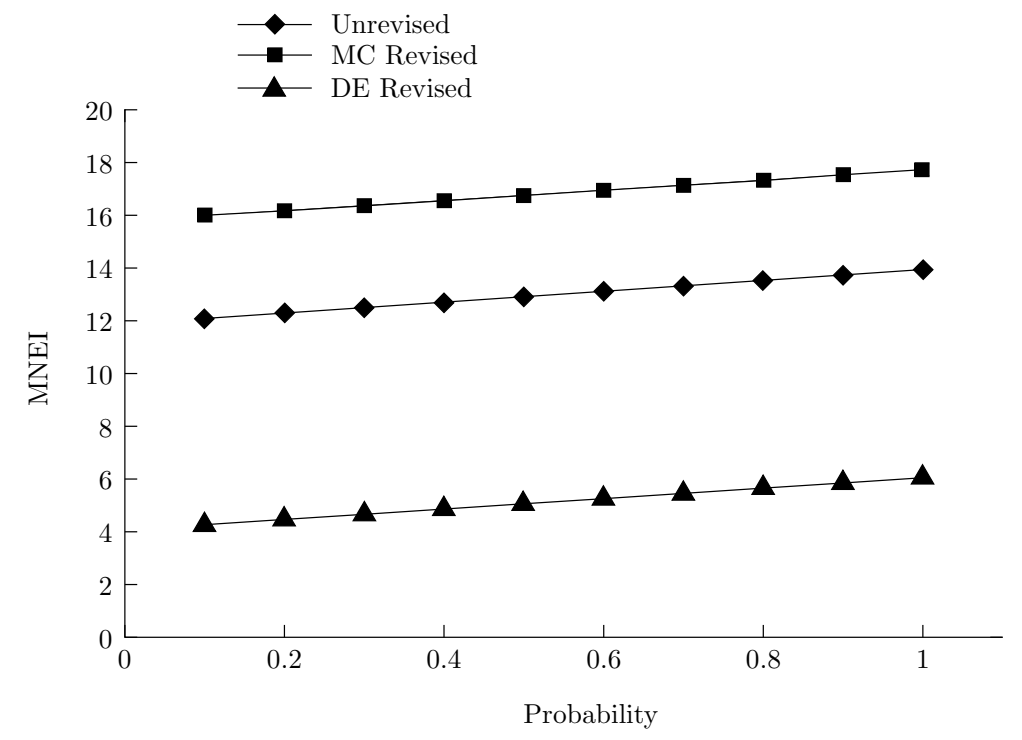

Figure 6: Relationship between MNEI and probability for the $10 \times 10$ system.

The results of the experimentation generally show that in an asymptotic case, the ranking of interdependent events depends on the technique used for revising events' marginal probabilities. Moreover, the Monte Carlo technique generates a world view closer to the one of the decision makers, while the world view generated by difference equation technique differs from that of the decision makers. The synonymous patterns of the graphs of MNEI against probability suggest that marginal probabilities revision can be a useful process for ranking interdependent events.

\section{Conclusion}

The objective of this paper was to determine the effect of marginal probabilities revision on the ranking of interdependent events. Two methods based on Monte Carlo and difference equation approaches were separately used to conduct the marginal probabilities revision process. A CIA system was developed and used to conduct the experimentation to determine the effect of marginal probabilities revision on the ranking of interdependent events.

The results of the experimentation show that the ranking of interdependent events may depend quite significantly on the method used for revising the marginal probabilities of the 
events - the respective methods decidedly do not lead to equivalent or near-equivalent outcomes.

Moreover, in the present examples, the Monte Carlo method generated a world view closer to the initial one of the decision makers, while the world view generated by difference equation method differed quite substantially from that of the decision makers. This remained true, also when the original marginal probability of the most influential event was varied over a range of possible values.

However, one cannot at this stage conclude that one marginal probabilities revision method is better than the other. The difference equation method theoretically takes into account the influence of external forces which may impact on the selected events. This might be an important reason for the difference in the world views of the decision makers and that generated by the difference equation method. In the future, we hope to further investigate a marginal probabilities revision method that gives a better improvement of the world view of the decision makers.

In addition, we have started the process of eliciting the views of various stake-holders and decision makers with regard to drivers (events) of e-skills development in South Africa. To date more than 30 drivers have been proposed. These are being assessed in terms of their estimated probability of occurrence and their estimated impact on the e-skills development objective. The present study will inform the way in which we set about revising their initial probability estimates. It will also inform the sensitivity assessments of scenario planning exercises which are to be undertaken.

\section{References}

[1] AsAn SS \& ASAN U, 2007, Qualitative cross-impact analysis with time consideration, Technological Forecasting \& Social Change, 74(5), pp. 627-644.

[2] Chaо K, 2008, A new look at the cross-impact matrix and its applications in futures studies, Journal of Futures Studies, 12(4), pp. 45-52.

[3] Dalkey NC \& Helmer O, 1963, An experimental application of the Delphi method to the use of experts, Management Science, 9(3), pp. 458-467.

[4] Duval A, Fontela E \& Gabus A, 1975, Cross-impact analysis: A handbook on concepts and applications, pp. 202-222 in BALDWIN MM (ED), Portraits of complexity: Applications of systems methodologies to societal problems, Battelle Memorial Institute, Columbus (OH).

[5] ENZER S, 1971, Delphi and cross-impact techniques: An effective combination for systematic futures analysis, Futures, 3(1), pp. 48-61.

[6] ENZER S, 1972, Cross-impact techniques in technology assessment, Futures, 4(1), pp. 30-51.

[7] ENZER S, 1980, INTERAX-An interactive model for studying future business environments: Part I, Technological Forecasting and Social Change, 17(2), pp. 141-159.

[8] ENZER S, 1981, Exploring long term business climates and strategies with INTERAX, Futures, 13(6), pp. $468-482$.

[9] Godet M, 1994, From anticipation to action: A handbook of strategic prospective, UNESCO Publishing, Paris.

[10] Gordon TJ \& HAYWARD H, 1968, Initial experiments with the cross-impact matrix method of forecasting, Futures, 1(2), pp. 100-116.

[11] Li C, Sun Y \& Du Y, 2008, A new MCDM approach based on cross-impact analysis for ranking dependent alternatives, $4^{\text {th }}$ International Conference on Wireless Communications, Networking and Mobile Computing, Dalian, pp. 1-4. 
[12] Nguyen MT \& Dunn M, 2009, Some methods for scenario analysis in defence strategic planning, Technical Report Series DSTO-TR-2242, Defence Science and Technology Organization, Australia.

[13] Schlange LE \& JütTner U, 1997, Helping managers to identify the key strategic issues, Long Range Planning, 30(5), pp. 777-786.

[14] Turoff M, 1972, An alternative approach to cross-impact analysis, Technological Forecasting and Social Change, 3, pp. 309-339. 
